\title{
A Comparative Study and Analysis of EZW and SPIHT methods for Wavelet based Image Compression
}

\author{
CHETAN R. DUDHAGARA ${ }^{1}$ and MAYUR M. PATEL ${ }^{2}$ \\ ${ }^{1,2}$ Computer Science Department Natubhai V. Patel College of Pure and Applied \\ Sciences Vallabh Vidyanagar, Anand, Gujarat, India.
}

\begin{abstract}
In recent years there has been widely increase the use of digital media everywhere. To increase the use of digital media, there is a huge problem of storage, manipulation and transmission of data over the internet. These digital media such as image, audio and video require large memory space. So it is necessary to compress the digital data to require less memory space and less bandwidth to transmission of data over network. Image compressions techniques are used to compress the data for reduce the storage requirement. It plays an important role for transfer of data such as image over the network. Two methods are used in this paper on Barbara image. This compression study is performed by using Set Partitioning In Hierarchical Trees (SPIHT) and Embedded Zero tree Wavelet (EZW) compression techniques. There are many parameters are used to compare this techniques. Mean Square Error (MSE), Pick Signal to Noise Ration (PSNR) and Compression Ratio (CR) are used at different level of decompositions.
\end{abstract}

Article History
Received: 17
Accepted:09 A
Keywords
Transmission,
Bandwidth,
Wavelet,
SPIHT,
EZW,
PSNR,
MSE,
CR.

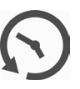

\section{Article History}

Received: 17 July 2017

Accepted:09 August 2017

\section{Keywords}

Transmission

Wavelet,

SPIHT,

EZW,

\section{Introduction}

Digital image is an array which contains many pixel values. The neighbor pixels may be correlated to each other pixel so it contains redundant bits. These redundant bits are removed from the images for reduce the size of image by using different compression algorithms. There are two components : redundancy reduction and irrelevant reduction in image compression. The less important data will remove in irrelevant reduction. This removed information was not received by receiver also. There are three types of redundancies. It includes coding; inter pixel and psycho visual redundancy. Where less number of code words is required, it used coding redundancy. Where correlation of different pixels are available in an image, it used inter pixel redundancy. The information which is ignored by human visual system is called Psycho visual redundancy.

CONTACT DR. CHETAN R. DUDHAGARA crdudhagara32@gmail.com 9 Computer Science Department Natubhai V. Patel College of Pure and Applied Sciences Vallabh Vidyanagar, Anand, Gujarat, India.

(C) 2017 The Author(s). Published by Enviro Research Publishers

This is an $\mathbf{6}$ Open Access article licensed under a Creative Commons Attribution-NonCommercial-ShareAlike 4.0 International License (https://creativecommons.org/licenses/by-nc-sa/4.0/), which permits unrestricted NonCommercial use, distribution, and reproduction in any medium, provided the original work is properly cited.

To link to this article: http://dx.doi.org/10.13005/ojcst/10.03.17 


\section{Compression Techniques}

Image compression is key technology used for transmission of huge amount of real time data such as image, audio and video on limited bandwidth network. It is necessary to reduce the size of data before the send over network. Wavelet based transformation is widely used techniques. For images, the widely using coding algorithms are based on wavelet transform include the set partitioning in hierarchical trees (SPIHT), the embedded zero tree wavelet (EZW), the wavelet difference reduction (WDR) algorithms.

\section{EZW}

EZW stands for Embedded Zero-tree Wavelet. It gives good compression result on different test images. It was introduced by J. Shapiro. It is lossless compression algorithm it done through adaptive arithmetic coding. It is a valuable image compression algorithm. It produces a fully embedded bit stream for image coding. There are four concepts in this method such as DWT, absent of significant information, entropy coding, lossless compression through arithmetic coding. or near to zero. It near to zero because mostly all standard test images has low frequency information. High quality scheme is used for high frequency information. This is play important role in human perception of image quality.

\section{SPIHT}

SPIHT stands for Set Partitioning In Hierarchical Tree. This is modified version of EZW method. It is wavelet based image compression coder. It convert image to wavelet transform. The most important features of this algorithm are it produced the best quality of image with high PSNR values.

It can be used for lossless image compression and it gives fully progressive bit stream. The main advantage of this algorithm is that it is fully progressive. This method codes wavelet transform coefficients and send the bits. So it helps to regenerate the original copy of image progressively. This image compression method is very efficient. It has fewer complexes, and it generates compressed bit-stream which is help to efficiently decoded at different data rates.

When the bit rate is low, mostly coefficients produced by a sub band transform will be zero

The following figure shows a compression process of images using SPIHT.

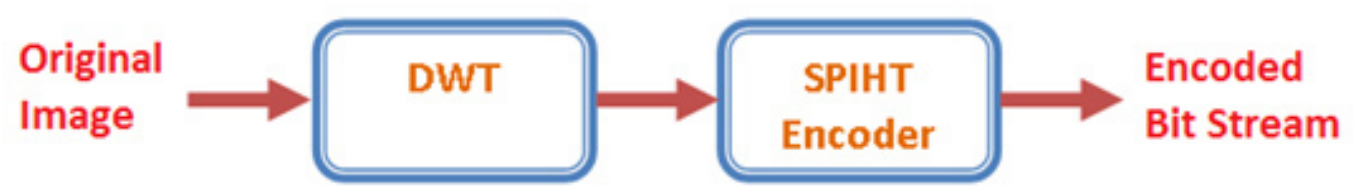

Fig. 1: Block Diagram of Image Compression

The compression consists of two parts :

- $\quad$ DWT : Discrete Wavelet Transform

- $\quad$ SPIHT Encoder : Encoder for image compression
The following figure shows a decompression process of image using SPIHT. It is invert process of compression.

The decompression consists of two parts :

- $\quad$ SPIHT Decoder : Decoder for information reconstruction

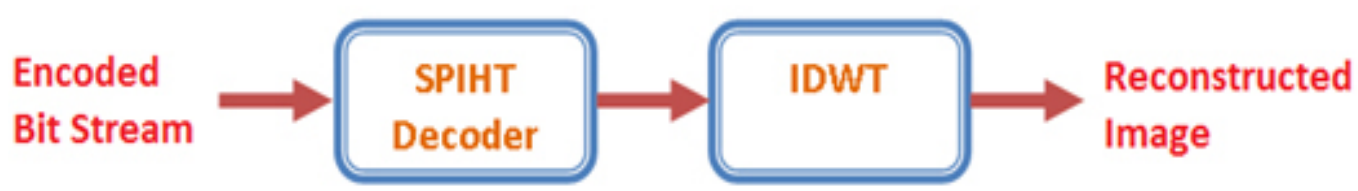

Fig. 2: Block Diagram of Image Decompression 


\section{- IDWT : Inverse Discrete Wavelet Transform}

The SPIHT decoder reconstructs the decomposed image which is proposed by the IDWT.

\section{Performance Criterion}

We can measure the performance of image compression methods by Mean Square Error (MSE), Peak Signal to Noise Ratio (PSNR),
Compression Ratio (CR) and elapsed time for the image compression.

\section{Experimental Study}

In this paper the stu;dy is performed on Barbara.jpg image by using wavelet based image compression method. This study is performed using SPIHT and EZW methods of Haar wavelet based compression at different level of decomposition. In this paper we perform the study up to five decomposition levels.
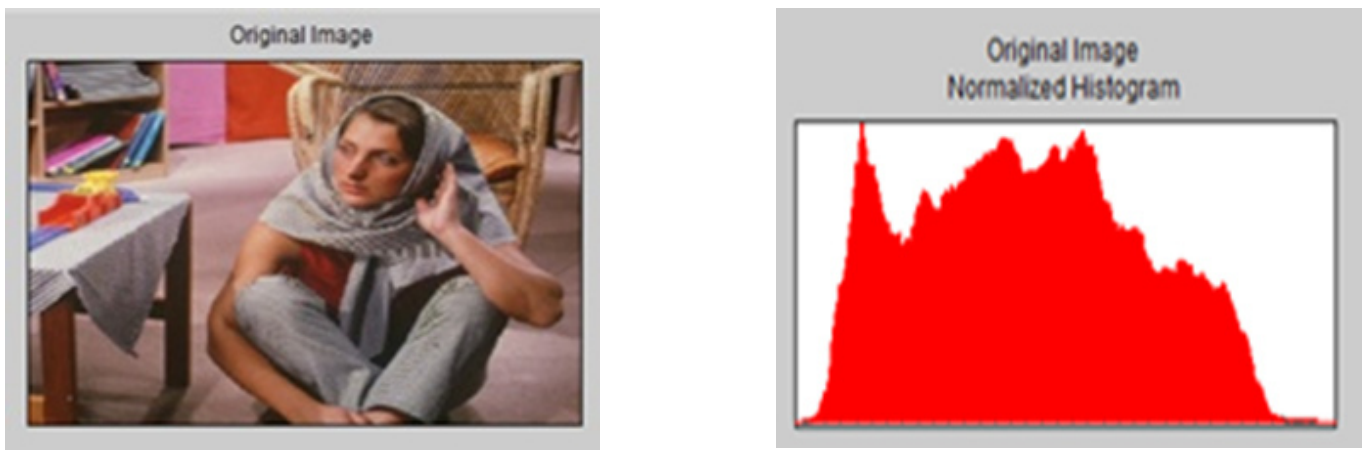

Fig. 3: Original Image with Histogram

1

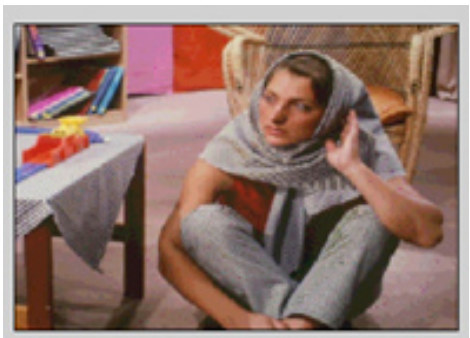

Comoressed Image

2
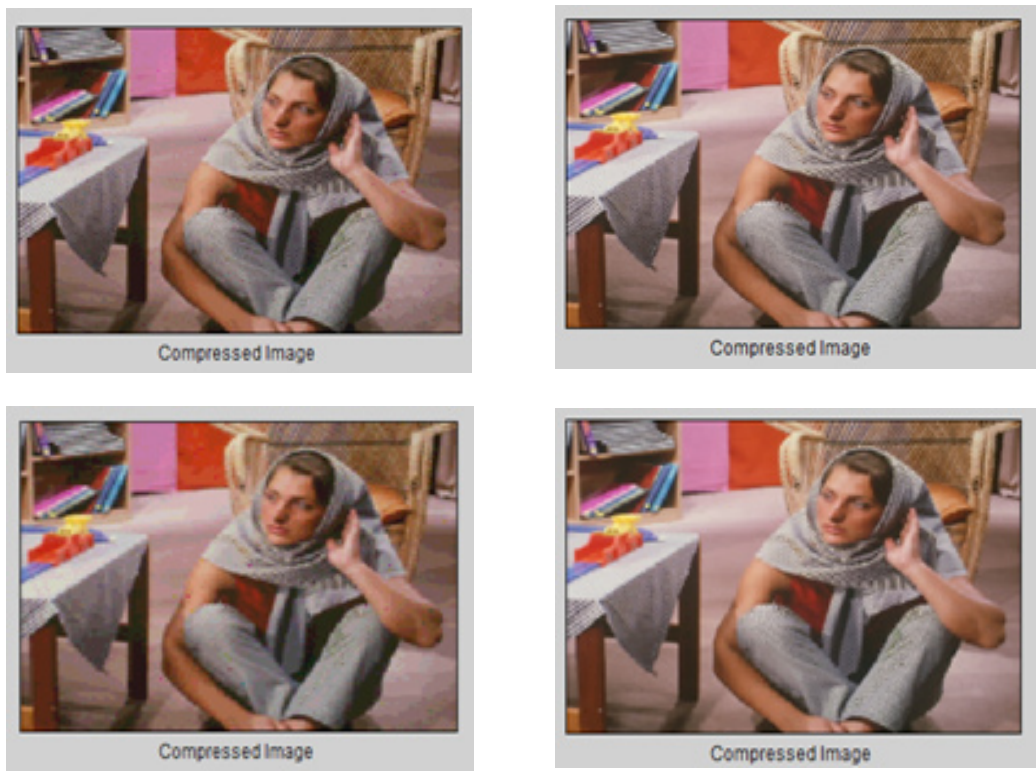
3

4

5
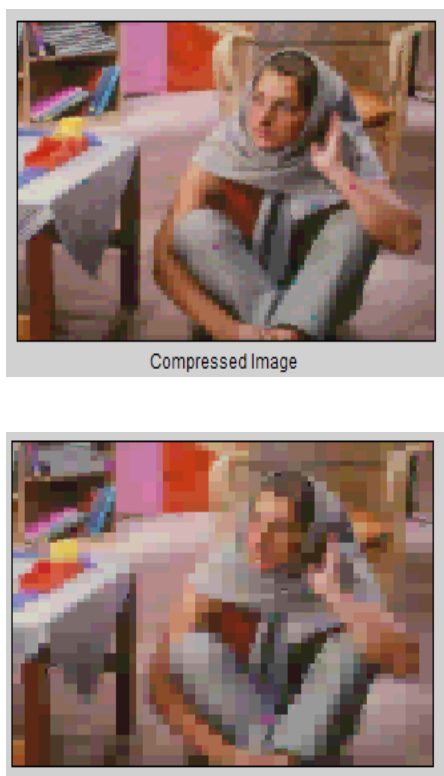

Compressed Image

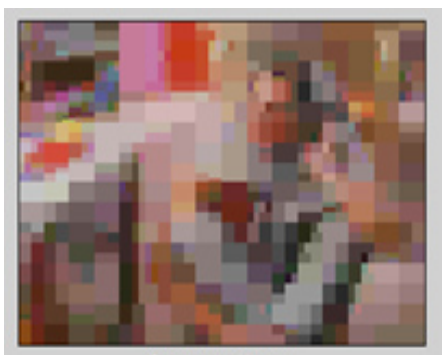

Compressed Image
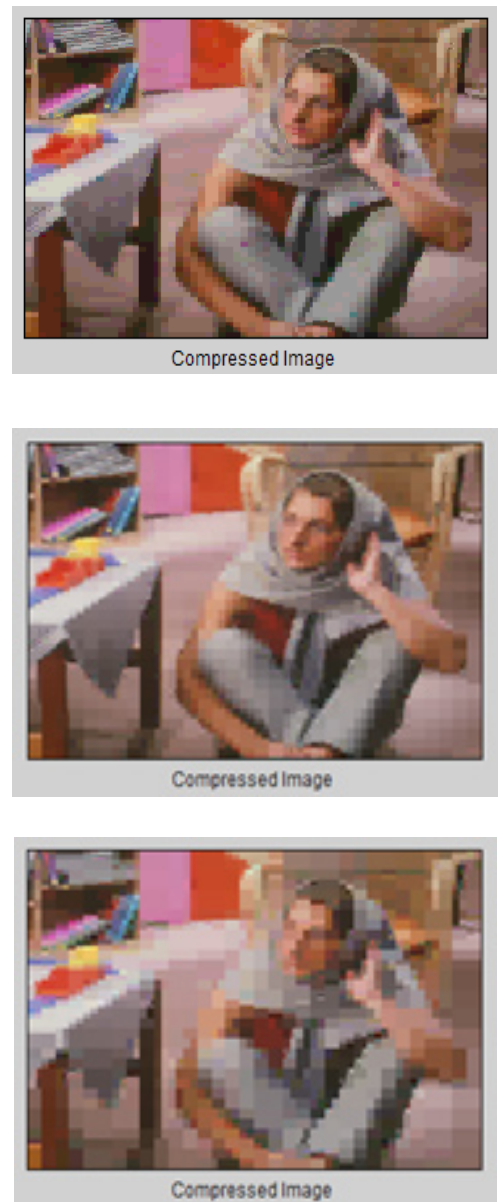

Compressed image

\begin{tabular}{lcccccc}
\hline \multirow{2}{*}{$\begin{array}{l}\text { Decomposition } \\
\text { Level }\end{array}$} & \multicolumn{3}{c}{ EZW } & \multicolumn{3}{c}{ SPIHT } \\
\cline { 2 - 7 } & MSE & PSNR & CR (\%) & MSE & PSNR & CR (\%) \\
\hline $\mathbf{1}$ & 16.44 & 35.97 & 35.53 & 137.7 & 26.74 & 65.03 \\
$\mathbf{2}$ & 64.94 & 30.01 & 12.16 & 226.2 & 24.59 & 16.89 \\
$\mathbf{3}$ & 155.1 & 26.23 & 3.83 & 344.4 & 22.76 & 4.44 \\
$\mathbf{4}$ & 276.0 & 23.72 & 1.24 & 511.0 & 21.05 & 1.22 \\
$\mathbf{5}$ & 437.6 & 21.72 & 0.45 & 765.5 & 19.29 & 0.35 \\
\hline
\end{tabular}

\section{Performance Analysis And Result Discusion}

Below table shows the different wavelet based image compression algorithm at different decomposition level on Barbara image.

\section{Conclusion}

The main objective of this comparative study is to compress an image using two different methods and study the comparative results. In this study we take image of Barbara. This original image we 
converted into compressed image using EZW and SPIHT methods. In this study comparative analysis of EZW and SPIHT is performed on the basis of two main parameters such as MSE and PSNR. This analysis and study present that SPIHT gives very good results to comparison with EZW methods. At the end of conclusion the compression ratio of SPIHT is high as compare with EZW methods.

\section{References}

1 Kaleka, Jashanbir Singh, Sharma Reecha., "Comparative performance analysis of Haar, Symlets and Bior wavelets on image compression using Discrete wavelet Transform", International journal of Computers and Distributed Systems, Volume 1, Issue 2, August,2012.

2 Chandandeep Kaur, Sumit Budhiraja, "Improvements of SPIHT in Image Compression- Survey", International Journal of Emerging Technology and Advanced Engineering, Vol. 3, Issue 1, January 2013. Sandeep Kaur, Garanpreet Kaur, Dheerendra Singh, "Comparative Analysis of Haar and Coiflet Wavelets using Discrete Wavelet Transform in Digital Image Compression, International Journal of Engineering Research and Applications Vol. 3, Issue 3, May-Jun 2013.

4 Monika Rathee, Alka Vij, "Image compression Using Discrete Haar Wavelet Transforms", International Journal of Engineering and Innovative Technology (IJEIT), Volume $\mathbf{3}$, Issue 12, June 2014. "Performance evaluation on EZW and SPIH image compression techniques", international Journal of Scientific and Research Publications, Vol. 4, Issue 10,

October 2014.

6 S. NirmalRaj, "SPIHT: A Set Partitioning in Hierarchical Trees Algorithm for Image Compression", Contemporary Engineering Sciences, Vol. 8, 2015.

7 P. Sunitha, J. L. Srinivas, "Performance Analysis of SPIHT algorithm in Image Compression", International Journal of Science, Engineering and Technology Research (IJSETR), Volume 4, Issue 1, January 2015.

8 Pooja Rawat, Arti Rawat, Swati Chamoli, "Analysis and Comparison of EZW, SPIHT and EBCOT Coding Schemes with Reduced Execution Time", International Journal of Computer Applications, Volume 130, No. 2, November 2015.

9 Bhagyashree I. Kochi, B. B. S. Kumar, "EZW and SPIHT Algorithms for Image Compression and Denoising", ITSI Transactions on Electrical and Electronics Engineering (ITSI-TEEE), Volume -4, Issue $-2,2016$.

10 Garima Singh, Pushpa Korange, Dikendra Verma, "Analysis of EZW and SPIHT Algorithms for Compression of an Image", International Journal on Emerging Technologies, Special Issue, 2017. 